\title{
Stabilization and Voltage Regulation of the Buck DC-DC Converter Using Model Predictive of Laguerre Functions
}

\author{
Chala Merga ABDISSA ${ }^{1}$, Kil To CHONG ${ }^{2 *}$ \\ ${ }^{1}$ Jeonbuk National University, \\ Baekje-daero 567 Deokjin-gu, Jeonju, 561-756, South Korea. \\ chalmer.abdissa@gmail.com \\ ${ }_{2}^{2}$ Jeonbuk National University, Baekje-daero 567 Deokjin-gu, Jeonju, 561-756, South Korea. \\ kitchong@jbnu.ac.kr, ('Corresponding author)
}

\begin{abstract}
This paper proposes a solution to stability and voltage regulation of switched mode buck DC-DC converter using a model predictive controller (MPC) of Laguerre functions. The MPC is used to compute the optimal control actions subject to constraints. To have a low computation burden and to avoid ill-conditioning, particularly for large prediction horizon, an exponentially weighted Laguerre based model predictive control (LMPC) is used. In order to validate the effectiveness of the proposed scheme, the performance of the proposed controller is compared with a linear quadratic regulator and classical linear state space MPC in MATLAB. Obtained results of simulation show that optimal voltage regulation has been achieved.
\end{abstract}

Keywords: Model predictive control, Optimal control, Ill-conditioning, Prediction horizon, Exponentially weighted, Laguerre, Linear quadratic regulator.

\section{Introduction}

The technological advance in the field of power electronics has increased the use of DC-DC power converters in a wide range of applications. Thus, their major role is to bring the voltage to the proper level, providing a regulated output voltage based on a supply voltage that can vary. Nevertheless, the control problems that are related to such converters still pose theoretical challenges. Usually, the control design for these switched systems is based on a continuous or discrete statespace averaged model [1]. For most of DC-DC power converters, the averaged model has to be locally linearized around a specific operating point because of the product between the state vector and the control input. A classic approach is to derive linear control laws based on an averaged linearized model: proportional-integral (PI) controllers [2], [3] state-feedback and Linear Quadratic Regulators (LQR) [4].

While PID controllers have wide application in many control problems, and often perform well without any modification or only with coarse tuning, they can operate badly in some applications, and do not in general perform optimally [5]. LQR is a relatively modern control technique that is effective but restricted to applications related with linear system models. In addition, the solution to the LQR problem, i.e., infinite prediction horizon, is available only in the unconstrained case, whereas MPC employs a finite prediction horizon to make the control problem tractable numerically.
An established solution to constraint handling in process control and in control of other relatively slow systems is MPC [6]. Just as LQR, MPC employs the model of the system to make predictions on future behavior of the system and optimize the control action accordingly. The reason for the slow speed of MPC its huge computational burden. To overcome this drawback, MPC with orthonormal basis function called Laguerre function [7] was proposed. The proposed MPC lowers computational burden significantly which makes it more suitable for real time implementation. In addition, an exponential data weighting is used to minimize numerical issue in MPC with large prediction horizon [8].

In this paper, the buck converter dynamics is reformulated to address the nonlinearity and is described in state space averaging (SSA) model. MPC employing [6], [7] is proposed to stabilize and regulate the output voltage of the buck DC-DC converter with systems constraints. The constraints are introduced from the converter circuitry. In particular, the control variable (duty cycle) is limited between zero and one. Additional constraints are imposed for safety measure such as the limit on inductor current. To prove the effectiveness of the suggested method, time-based simulations are carried out and compared with Optimal Discrete Linear Regulator (DLQR) and with the more common state space approach presented in [15]. The obtained results proved that the Laguerre based MPC (LMPC) is able to control successfully the buck converter in the transient and steady state. 


\section{Modeling the buck converter}

\subsection{Continuous Time Model}

The circuit topology of the converter is shown in Figure 1 where $R_{o}$ is the output resistance which, $R_{c}$ is the equivalent series resistance of the capacitor, $R_{L}$ is the internal resistance of the inductor, $L$ and $\mathrm{C}$ represent the inductance and the capacitance of the low-pass filter of the converter, respectively, and $V_{s}$ is the input voltage. The semiconductor switches $S_{1}$ and $S_{2}$, are operated by a pulse sequence with constants switching frequency $f_{s}$ (with period $T_{s}$ ). The duty cycle $d$ is defined as $d=t_{\text {on }} / T_{s}$ where $t_{\text {on }}$ represents the interval within the switching period during which the $S_{I}$ switch is closed.

We consider the state vector

$$
x_{m}(t)=\left[\begin{array}{ll}
I_{L}(t) & V_{o}(t)
\end{array}\right]^{T}
$$

The set of continuous-time state space equations describing the converter's behavior of the two configurations in CCM (Continuous Conduction Mode) are:

$$
\begin{aligned}
& \dot{x}_{m}(t) \\
& =\left\{\begin{array}{l}
A_{o n} x(t)+B_{o n} V_{s}, k T_{s} \leq t<(k+d(k)) T_{s} \\
A_{\text {off }} x(t),(k+d(k)) T_{s} \leq t<(k+1) T_{s}
\end{array}\right. \\
& y(t)=C x(t)
\end{aligned}
$$

where the matrices $\mathrm{A}_{\text {on }}, \mathrm{B}_{\text {on }}$ and $\mathrm{C}$ are given by

$$
A_{o n}=A_{o f f}=\left[\begin{array}{cc}
-\frac{R_{l}}{L} & -\frac{1}{L} \\
\frac{R_{o}}{C\left(R_{o}+R_{c}\right)}\left(1-R_{c} R_{l} \frac{C}{L}\right) & -\frac{1}{C\left(R_{o}+R_{c}\right)}\left(1+\frac{R_{c} R_{o} C}{L}\right)
\end{array}\right],
$$$$
B_{o n}=\left[\begin{array}{c}
\frac{1}{L} \\
\frac{R_{c} R_{o}}{\left(R_{c}+R_{o}\right) L}
\end{array}\right], C=\left[\begin{array}{ll}
1 & 0 \\
0 & 1
\end{array}\right]
$$

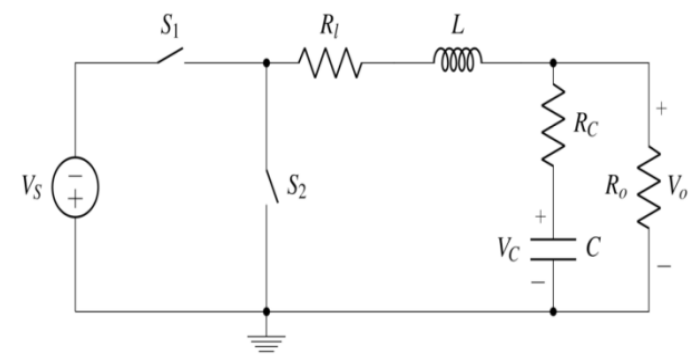

Figure 1. Buck Converter Topology
The continuous-time averaged model is

$$
\begin{aligned}
\dot{x}_{m}(t) & =\underbrace{\left[A_{o n} d+A_{o f f}(1-d)\right]}_{A_{m}} x_{m}(t)+\underbrace{B_{o n}}_{B_{m}} d V_{s} \\
& =A_{m} x(t)+B_{m} d V_{s} \\
y_{m}(t) & =\underbrace{[C d+C(1-d)]}_{C_{m}} x_{m}(t)=C_{m} x_{m}(t)
\end{aligned}
$$

In the following section, we derive a model to serve as prediction model for the optimal control problem formulation. For this, we reformulate the converter model.

\subsection{Reformulated Continuous Time Model}

We will be motivated to remove $\mathrm{V}_{\mathrm{s}}$ from the model equations by using it to scale the physical quantities (states and output voltage reference) used in the model. Hence, we introduce the state $x_{m}^{\prime}(t)=\frac{x_{m}(t)}{V_{s}}$ to scale (4) and (5). This yields the reformulated state space equations

$$
\begin{aligned}
& \dot{x}_{m}^{\prime}(t)=A_{m} x^{\prime}(t)+B_{m} u(t) \\
& y_{m}^{\prime}(t)=C_{m} x_{m}^{\prime}(t)
\end{aligned}
$$

where the matrices $A_{m}, B_{m}$ and $C_{m}$ are as in (4) and (5). Hereafter, we use control the notation for input signal, $\mathrm{u}(\mathrm{t})$ and duty cycle, $\mathrm{d}(\mathrm{t})$ interchangeably as required.

Since in real time operation, the input voltage is either piecewise constant or varies only slowly compared to the fast switching frequency, the normalized converter can serve as a sufficiently accurate prediction model. Since normalizing makes the prediction model equations not influenced by (the time-varying) input voltage $V_{s}$, the matrices, $A_{m}, B_{m}$ and $C_{\mathrm{m}}$ in (4) and (5) are time-invariant. Thus, the only time-varying model parameters are the scaled output voltage reference $v_{o, \text { ref }}$ and normalized current limit $\left(i_{L, \text { max }} i_{L, \text { min }}\right)$.

\subsection{Discretization of the continuous time model}

This paper is based on discrete model based MPC and hence (7) is discretized at sampling period of $T_{s}$ using a zero-order hold technique, yielding the discrete state-space model $x_{m}^{\prime}(k+1)=A_{d} x_{m}^{\prime}(k)+B_{d} u(k)$

$y_{m}^{\prime}(k)=C_{d} x_{m}^{\prime}(k)$ 
where $A_{d}=e^{A_{m} T_{s}}, B_{d}=\int_{0}^{T_{s}} e^{A_{m} T_{s}} B_{m} d \tau, C_{d}=C_{m}$

Let $\Delta x_{m}^{\prime}(k)=x_{m}^{\prime}(k)-x_{m}^{\prime}(k-1)$ and $\Delta u(k)=u(k)-u(k-1)$ denote the incremental state and input vectors, respectively, computed from the corresponding vectors in (8).

The state dynamics in the incremental model

$\Delta x_{m}^{\prime}(k+1)=A_{d} \Delta x_{m}^{\prime}(k)+B_{d} \Delta u(k)$

In a similar manner the output incremental dynamics are given by

$$
\begin{aligned}
y_{m}^{\prime}(k+1) & =y_{m}^{\prime}(k)+C_{d} A_{d} \Delta x_{m}^{\prime}(k) \\
& +C_{d} B_{d} \Delta u(k)
\end{aligned}
$$

By choosing a new state vector $x^{\prime}(k)=\left[\begin{array}{ll}\Delta x_{m}^{\prime}(k) & y_{m}^{\prime}(k)\end{array}\right]^{T}$ the augmented state-space model is obtained by combining (9) with (10).

$x^{\prime}(k+1)=A x^{\prime}(k)+B \Delta u(k)$

$y^{\prime}(k)=C x^{\prime}(k)$

where $A=\left[\begin{array}{cc}A_{d} & 0 \\ C_{d} A_{d} & I\end{array}\right], B=\left[\begin{array}{c}B_{d} \\ C_{d} B_{d}\end{array}\right], C=\left[\begin{array}{ll}0 & I\end{array}\right]$ and the $0_{l}, 0_{2}$ and $I$, respectively, are the zero and identity matrices of compatible dimensions.

\section{Model predictive control design}

To get high control performance while respecting state and input constraints we have used a model predictive control (MPC) approach. MPC provides a systematic way of handling constraint optimal control problems. In this and following sections the performance of a DC-DC controller designed using Laguerre-based MPC (LMPC) is compared with that designed using the more common linear state-space method (SS-MPC) as suggested in [15].

\subsection{Linear State-Space MPC (SS-MPC)}

Linear MPC, as unlike the usual forms of MPC, is attractive because the plant is modelled using a linear state space and plant constraints are modelled using linear equalities and inequalities. Using the augmented state-space model (13), the future state variables are calculated sequentially leading to the following equations:

$$
\begin{aligned}
& x(k+1 \mid k)=A x(k)+B \Delta u(k) \\
& x(k+2 \mid k)=A^{2} x(k)+A B \Delta u(k)+B \Delta u(k+1) \\
& \vdots \\
& x(k+N p \mid k)=A^{N_{p}} x(k)+A^{N_{p}-1} B \Delta u(k) \\
& +\ldots+A^{N_{p}-N_{c}} B \Delta u\left(k+N_{c}-1\right)
\end{aligned}
$$

where $N_{p}$ and $N_{c}$ are termed the prediction and control horizons, respectively.

The predicted output variables for the next $N p$ samples can be expressed in the compact form as

$$
\begin{aligned}
& Y=F x(k)+\Phi \Delta U \\
& Y=\left[\begin{array}{cccc}
y(k+1) & y(k+2) & \ldots & y\left(k+N_{p}\right)
\end{array}\right]^{T} \\
& \Delta U=\left[\begin{array}{llll}
\Delta u(k) & \Delta u(k+1) & \ldots & \Delta u\left(k+N_{c}-1\right)
\end{array}\right]^{T} \\
& F=\left[\begin{array}{c}
C A \\
C A^{2} \\
\vdots \\
C A^{N_{p}}
\end{array}\right] ; \Phi=\left[\begin{array}{cccc}
C B & 0 & \ldots & 0 \\
C A B & C B & \ldots & 0 \\
C A^{2} B & C A B & \ldots & 0 \\
\vdots & \vdots & \vdots & \vdots \\
C A^{N_{P}-1} B & C A^{N_{p}-2} B & \ldots & C A^{N_{p}-N_{c}} B
\end{array}\right]
\end{aligned}
$$

Then cost function used for the MPC is

$J=\left(R_{s}-Y\right)^{T} \bar{Q}\left(R_{s}-Y\right)+\Delta U^{T} \bar{R} \Delta U$

where

$$
\bar{Q}=\operatorname{diag}[Q, \ldots, Q] \text { and }
$$

$\bar{R}=\operatorname{diag}[R, \ldots, R]$ are block diagonal matrices that have identical component matrices $Q$ and $R$ respectively. Here, $Q$ is positive semidefinite matrix and $\mathrm{R}$ is a positive definite matrix. $R_{s}$ is the reference signal vector.

Minimizing the cost function (16) yields the optimal control vector

$$
\Delta U=\left(\Phi^{T} \bar{Q} \Phi+\bar{R}\right)^{-1} \Phi^{T} \bar{Q}\left(R_{s}-F x(k)\right)
$$

and

applying receding horizon principle

$$
\Delta u(k)=\left[\begin{array}{llll}
I & 0 & \cdots & 0
\end{array}\right] \Delta U
$$

\subsection{Laguerre Based Model Predictive Control (LMPC)}

MPC can be designed using orthonormal functions wherein the control signal $\Delta U$ is represented using Laguerre functions. Since the state and output signals can also be expressed in terms of $\Delta U$, and then they too can be expressed using Laguerre functions. 
In this paper, orthonormal basis Laguerre function [7] is used for modeling control signal of (17). The $z$-transform of $j$ th Laguerre function is given by

$$
\Gamma_{j}=\frac{\sqrt{1-a^{2}}}{z-a}\left[\frac{1-a z}{z-a}\right]^{j-1}
$$

where $0 \leq a \leq 1$ is the pole of Laguerre polynomial.

The control input variable can be expressed by the following Laguerre functions:

$\Delta u\left(k_{i}+k\right) \approx \sum_{j=1}^{N} c_{j}\left(k_{i}\right) 1_{\mathrm{j}}\left(k_{i}\right)$

where $l_{j}$ is the inverse $z$-transform of $\Gamma_{j}$ in the discrete domain. The coefficients $c_{j}$ are unknowns and must be acquired from systems data. The parameters $a$ and $N$ are used for tuning and can be adjusted by accordingly. Generally, choosing larger value for $N$ increases the accuracy of input sequence estimation [7] and the control horizon $\left(N_{c}\right)$ is related to the parameters $a$ and $N$ [14] by

$a \approx e^{-N / N_{c}}$

Equation (19) can be rewritten as

$$
\Delta u\left(k_{i}+k\right)=L(k)^{T} \eta
$$

$$
L(k)=\left[\begin{array}{llll}
l_{1}(k) & l_{2}(k) & \ldots & l_{N}(k)
\end{array}\right]^{\mathrm{T}}, \eta=\left[\begin{array}{llll}
c_{1} & c_{2} & \ldots & c_{N}
\end{array}\right]^{\mathrm{T}}
$$

At an arbitrary future instant $m$, the state is described using Laguerre functions as

$$
x\left(k_{i}+m \mid k_{i}\right)=A^{m} x\left(k_{i}\right)+\sum_{i=0}^{m-1} A^{m-i-1} B L(i)^{T} \eta
$$

Similarly, the output is described as

$$
y\left(k_{i}+m \mid k_{i}\right)=C A^{m} x\left(k_{i}\right)+\sum_{i=0}^{m-1} C A^{m-i-1} B L(i)^{T} \eta
$$

The objective is to come up with the coefficient vector $\eta$ that minimizes the cost function

$$
\begin{aligned}
J & =\sum_{m=1}^{N_{P}} x\left(k_{i}+m \mid k_{\mathrm{i}}\right)^{\mathrm{T}} Q x\left(k_{i}+m \mid k_{\mathrm{i}}\right) \\
& +\eta^{\mathrm{T}} R_{L} \eta
\end{aligned}
$$

where $Q \geq 0$ and $R_{L} \geq 0$ is the weighting matrices for tuning purpose.
Equation (24) can be rewritten as

$x\left(k_{i}+m \mid k_{i}\right)=A^{m} x\left(k_{i}\right)+\Phi(m)^{T} \eta$

where $\Phi(m)^{T}=\sum_{i=0}^{m-1} A^{m-i-1} B L(i)^{T}$

By substituting (25) in (24) and minimizing $\mathrm{J}$ $(\partial J / \partial \eta=0)$, the Laguerre coefficients vector $\eta=-\Omega^{-1} \Psi x\left(k_{i}\right)$

with $\Omega=\sum_{m=1}^{N p} \Phi(m) Q \Phi(m)^{T}+R_{L}, \Psi=\Phi(m) Q A^{m}$

The objective function (24) is subjected to the constraints on input and output variable that can be defined in the following form

$u_{\min } \leq u\left(k_{i}+m\right) \leq u_{\max }$

$\Delta u_{\min } \leq \Delta u\left(k_{i}+m\right) \leq \Delta u_{\max }$

$y_{\min } \leq y\left(k_{i}+m\right) \leq y_{\text {max }}$

where $\Delta u\left(k_{i}+m\right)=L(m)^{T} \eta, u_{\min }$ and $u_{\max }$ are lower and upper limits on control variable, $y_{\min }$ and $y_{\max }$ are lower and upper limits on the output variables and $\Delta u_{\min }$ and $\Delta u_{\max }$ lower and upper limits on incremental control variable respectively.

\subsection{Stability}

In practice, the choose of larger prediction horizon is limited by numerical problems, particularly in the process with high sampling rate. A wellestablished method to solve this problem is to use exponential data weighting in the objective function [12] an idea originally proposed by Anderson and Moore [13].

More specifically, we will focus on the discrete exponential factor $e^{\lambda \Delta t}$ for $t>0$ and the discrete weights forming a sequence $\left\{\alpha_{j}, j=0,1,2, \ldots\right\}$ in which we set $\alpha=e^{\lambda \Lambda t}$ with $\Delta t$ being the sampling interval.

The proposed objective function is equivalent the linear quadratic regulator (LQR) systems but with discrete weights included

$$
\begin{aligned}
\hat{J} & =\sum_{j=1}^{N_{P}} \alpha^{-2 j} x\left(k_{i}+j \mid k_{\mathrm{i}}\right)^{\mathrm{T}} Q x\left(k_{i}+j \mid k_{\mathrm{i}}\right) \\
& +\sum_{j=0}^{N_{p}} \alpha^{-2 j} \Delta u\left(k_{i}+j\right)^{T} R \Delta u\left(k_{i}+j\right)
\end{aligned}
$$


In compact form, the exponentially weighted cost function is

$$
\begin{aligned}
\hat{J} & =\sum_{j=1}^{N_{P}} \hat{x}\left(k_{i}+j \mid k_{\mathrm{i}}\right)^{\mathrm{T}} Q x\left(k_{i}+j \mid k_{\mathrm{i}}\right) \\
& +\sum_{k=0}^{N_{p}} \Delta \hat{u}\left(k_{i}+j\right)^{T} R \Delta \hat{u}\left(k_{i}+j\right)
\end{aligned}
$$

with state equation

$\hat{x}(k+1)=A_{\alpha} \hat{x}(k)+B_{\alpha} \Delta \hat{u}(k)$

and $A_{\alpha}=A / \alpha$ and $B_{\alpha}=B / \alpha$

with $Q \geq 0, R>0$, and $N_{p} \rightarrow \infty$, minimizing the cost function $\hat{J}$ is similar to the DLQR problem which is solved using algebraic Riccati equation (31). Then the state feedback control gain for the stabilization $\hat{K}$,

$\hat{K}=\left(R+\alpha^{-2} B^{T} \hat{P} B\right) \alpha^{-2} B^{T} \hat{P} A$

$\frac{A^{T}}{\alpha}\left[\hat{P}-\hat{P}-\frac{B}{\alpha}\left(R+\frac{B^{T}}{\alpha} \hat{P}-\frac{B}{\alpha}\right)^{-1} \frac{B^{T}}{\alpha} \hat{P}\right] \frac{A}{\alpha}+Q-\hat{P}=0$

which makes the closed loop system stable with all its eigenvalues inside the unit circle and the closed loop system being described by

$$
\hat{x}\left(k_{i}+j+1 \mid k_{i}\right)=\alpha^{-1}(A-B \hat{K}) \hat{x}\left(k_{i}+j \mid k_{i}\right)
$$

From (32), the modified system has all its eigenvalues inside the unit circle by increasing $\mathrm{N}_{p} \rightarrow \infty$. So

$\alpha^{-1}\left|\lambda_{\text {max }}(A-B \hat{K})\right|<1$

Thus, by choosing $\alpha>1$ it is possible to make stable. Several simulations on indicates the choice of $\alpha$ greater than unity stabilizes the system.

Table 1. Converter and controller parameters

\begin{tabular}{|l|l|l|l|}
\hline \multicolumn{4}{|c|}{ Converter Parameters } \\
\hline \multicolumn{2}{|c|}{ In S.I. } & \multicolumn{2}{c|}{ In p.u. } \\
\hline $\mathrm{L}$ & $27 \mu \mathrm{H}$ & $\mathrm{x}_{\mathrm{I}}$ & 0.6786 \\
\hline $\mathrm{C}$ & $4.7 \mu \mathrm{F}$ & $\mathrm{x}_{\mathrm{c}}$ & 11.8124 \\
\hline $\mathrm{R}_{\mathrm{c}}$ & $0.025 \Omega$ & $\mathrm{r}_{\mathrm{c}}$ & 0.0025 \\
\hline $\mathrm{R}_{\mathrm{L}}$ & $0.4 \Omega$ & $\mathrm{r}_{1}$ & 0.04 \\
\hline $\mathrm{R}_{0}$ & $10 \Omega$ & $\mathrm{r}_{\mathrm{o}}$ & 1 \\
\hline $\mathrm{V}_{\mathrm{s}}$ & $20 \mathrm{~V}$ & $\mathrm{~V}_{\mathrm{s}}$ & 1 \\
\hline $\mathrm{V}_{\text {oref }}$ & $10 \mathrm{~V}$ & $\mathrm{~V}_{\text {o,ref }}$ & 0.5 \\
\hline $\mathrm{I}_{\mathrm{L}, \max }$ & $3 \mathrm{~A}$ & $\mathrm{i}_{\mathrm{L}, \max }$ & 1.5 \\
\hline $\mathrm{V}_{0, \max }$ & $15 \mathrm{~V}$ & $\mathrm{~V}_{0, \max }$ & 0.75 \\
\hline
\end{tabular}

\begin{tabular}{|l|l|}
\hline \multicolumn{2}{|c|}{ Controller Parameters } \\
\hline $\mathrm{N}_{\mathrm{p}}$ & 200 \\
\hline $\mathrm{T}_{\mathrm{s}}$ & $25 \mu \mathrm{s}$ \\
\hline
\end{tabular}

\section{Simulation parameters and results}

Simulations using MATLAB have been done in order to prove the effectiveness of proposed method. Table 1 shows the parameters of the DC-DC buck converter used in simulation. The constraints on the states input and incremental control inputs are chosen so as to assure the keep the signals at physically appropriate values as follows:

$$
\begin{aligned}
d_{\text {min }} & =0 \leq d(t) \leq 1=d_{\max } \\
\Delta d_{\min } & =-0.5 \leq \Delta d(t) \leq 0.5=\Delta d_{\max } \\
y_{\text {min }} & =\left[\begin{array}{c}
i_{L \min } \\
v_{\text {omin }}
\end{array}\right]=\left[\begin{array}{l}
0 \\
0
\end{array}\right] \leq y(t) \\
& \leq\left[\begin{array}{c}
i_{L \max } \\
v_{\text {omax }}
\end{array}\right]=\left[\begin{array}{c}
1.5 \\
0.75
\end{array}\right]=y_{\max }
\end{aligned}
$$

\subsection{Use of Laguerre parameters}

Assuming we want to attain a control horizon of 10. To grant stability for large prediction horizon $\left(\mathrm{N}_{\mathrm{p}}=200\right)$, we used $\alpha=1.6$ and $\lambda=1$. The parameter $N$ is an integer to specify the network order. We used (20) to find the corresponding scaling factor $a$ as shown in Table 2 .

Table 2. Scaling factor $a$ for constant control horizon and varying $\mathrm{N}$

\begin{tabular}{|c|c|c|}
\hline $\mathbf{N}_{\mathbf{c}}$ & $\mathbf{N}$ & $\mathbf{a}$ \\
\hline 10 & 2 & 0.8187 \\
\hline 10 & 4 & 0.6703 \\
\hline 10 & 6 & 0.5488 \\
\hline 10 & 8 & 0.4493 \\
\hline 10 & 10 & 0.3679 \\
\hline
\end{tabular}

Two cases are considered in order to analyze the system's response for square signal profile of the output voltage reference $\mathrm{V}_{\mathrm{o}, \mathrm{ref}}: 10-5-10 \mathrm{~V}$. 
Case 1. Set the Laguerre Order $\mathrm{N}$ to be large value $(N=8)$. See Table 3 and Figure 2.
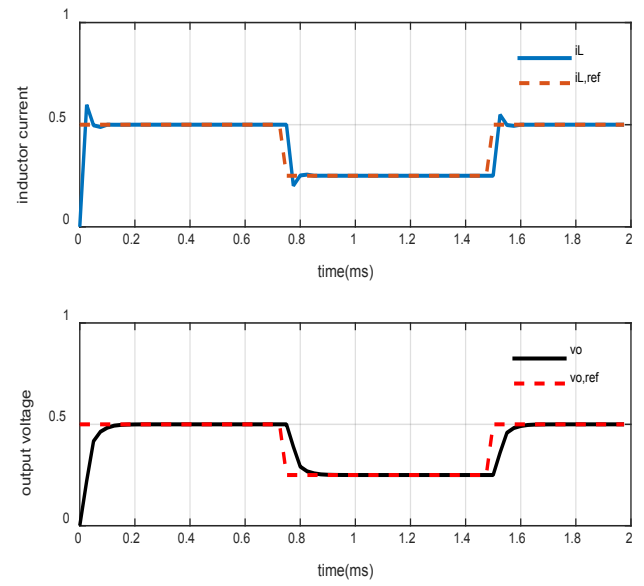

(a)
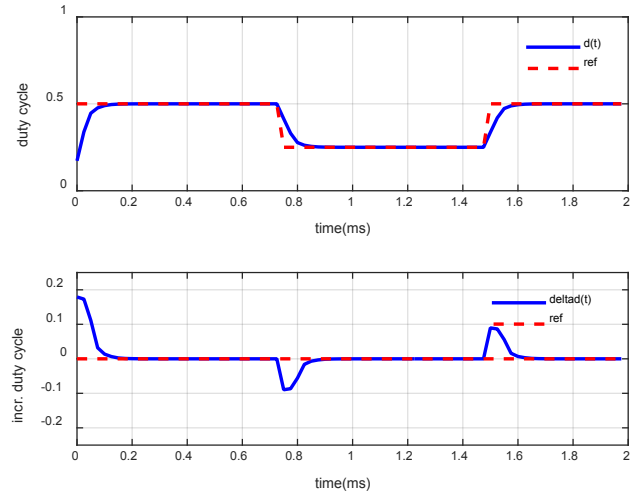

(b)

Figure 2. Simulation results for reference voltage variations $\mathrm{V}_{\mathrm{o}, \mathrm{ref}}: 10-5-10 \mathrm{~V}$

(a) Inductor current iL and Output voltage $v_{o}$ obtained using $\mathrm{LMPC}$ for $\mathrm{N}=8$

(b) Duty Cycle and incremental duty cycle obtained using LMPC for $\mathrm{N}=8$

Case 2. Set the parameter smaller value $(N=2)$.
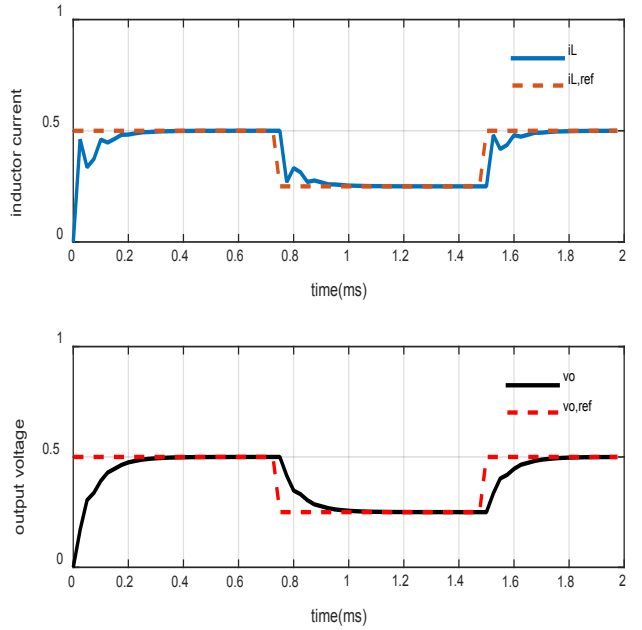

(a)
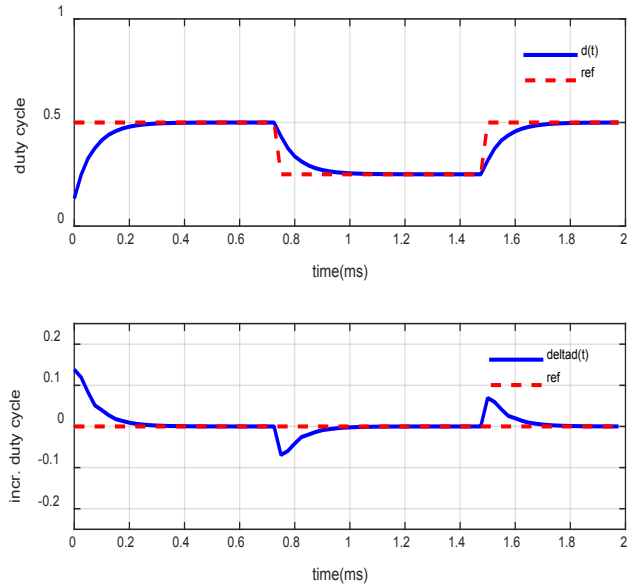

(b)

Figure 3. Simulation results for reference voltage variations $\mathrm{v}_{\mathrm{o}, \mathrm{ref}}: 10-5-10 \mathrm{~V}$

(a) Inductor current iL and Output voltage $\mathrm{v}_{\mathrm{o}}$ obtained using LMPC for $\mathrm{N}=2$

(b) Duty Cycle $\mathrm{d}(\mathrm{t})$ and incremental duty cycle $\Delta \mathrm{d}(\mathrm{t})$ obtained using LMPC for $\mathrm{N}=2$

It can be seen from Table 3 that the closed loop predictive control system is very similar to the DLQR system.

Table 3. Closed loop Eigen values and feedback gain vector when $\mathrm{N}=8$

\begin{tabular}{|l|c|c|c|}
\hline \multirow{2}{*}{$\begin{array}{c}\text { Performance } \\
\text { Metrics }\end{array}$} & \multicolumn{4}{|c|}{ Control Strategy } \\
\cline { 2 - 5 } & \multicolumn{4}{|c|}{ LMPC } & \multicolumn{1}{|c|}{ DLQR } \\
\hline Feedback gain & {$\left[\begin{array}{lllll}-0.0464 & -0.5118 & 0.1792 & 0.1792\end{array}\right]$} & {$\left[\begin{array}{llll}-0.0463 & -0.5118 & 0.1792 & 0.1792\end{array}\right]$} \\
\hline Eigen Values & $-0.1011 \pm \mathrm{j} 0.2268,1,0.4135$ & $-0.1012 \pm \mathrm{j}$ & $0.2267,1,0.2305$ \\
\hline
\end{tabular}

Table 4. Closed loop Eigen values and feedback gain vector when $\mathrm{N}=2$

\begin{tabular}{|c|c|c|}
\hline \multirow{2}{*}{$\begin{array}{c}\text { Performance } \\
\text { Metrics }\end{array}$} & \multicolumn{3}{|c|}{ Control Strategy } \\
\cline { 2 - 4 } & LMPC & \multicolumn{1}{|c|}{ DLQR } \\
\hline Feedback gain & {$[-0.0424-0.28600 .13840 .1384]$} & {$[-0.0463-0.51180 .17920 .1792]$} \\
\hline Eigen Values & $-0.2954 \pm \mathrm{j} 0.4628,1,0.6973$ & $-0.1012 \pm \mathrm{j} 0.2267,1,0.4135$ \\
\hline
\end{tabular}


Table 5. Performances comparison of comparison LMPC and SS-MPC obtained in simulation for reference voltage $\mathrm{V}_{\text {oref }}: 10-5-10 \mathrm{~V}$

\begin{tabular}{|c|c|c|}
\hline \multirow{2}{*}{$\begin{array}{c}\text { Performance } \\
\text { Metrics }\end{array}$} & \multicolumn{2}{|c|}{ Control Strategy } \\
\hline & LMPC & SS-MPC \\
\hline Feedback gain & {$\left[\begin{array}{llll}-0.1952 & -0.0725 & 0.1534 & 0.3200\end{array}\right]$} & {$\left[\begin{array}{lllll}-0.4012 & -0.1782 & 0.3774 & 0.5624\end{array}\right]$} \\
\hline Eigen Values & $-0.3749 \pm \mathrm{j} 0.4862,0,0.6888$ & $-0.3815 \pm 0.3798,0,0.4333$ \\
\hline Overshoot & $1.00 \%$ & $0.5008 \%$ \\
\hline Settling time ms & 1.5919 & 1.7081 \\
\hline
\end{tabular}

Table 6. Performances comparison of comparison LMPC and SS-MPC obtained in simulation for reference voltage $\mathrm{V}_{\text {oref }}: 10-5-10 \mathrm{~V}$

\begin{tabular}{|c|c|c|}
\hline \multirow{2}{*}{$\begin{array}{c}\text { Performance } \\
\text { Metrics }\end{array}$} & \multicolumn{2}{|c|}{ Control Strategy } \\
\hline & LMPC & SS-MPC \\
\hline Feedback gain & {$\left[\begin{array}{llll}0.3966 & -0.1715 & 0.3632 & 0.5584\end{array}\right]$} & {$\left[\begin{array}{llll}-0.4012 & -0.1782 & 0.3774 & 0.5624\end{array}\right]$} \\
\hline Eigen Values & $-0.3754 \pm \mathrm{j} 0.3887,0,0.4346$ & $-0.3815 \pm 0.3798,0,0.4333$ \\
\hline Overshoot & $1.00 \%$ & $1.00 \%$ \\
\hline Settling time ms & 1.5919 & 1.5919 \\
\hline
\end{tabular}

For the same simulation, (different instance), one can observe in Figure 2(b) and (3b) the evolution of the duty cycle, paired with its incremental value to allow for a direct comparison. In addition, because of the presence of exponentially decaying factor in the Laguerre functions, the increment on duty cycle is granted to converge to zero after the transient time.

Comparing the results in table 3 and 4 , it can be seen that the closed-loop control performance is more sensitive to the parameter $N$ when is small $(\mathrm{N}=2)$. This is very helpful in the situations when the optimal DLQR system does not gives us with satisfactory performance.

\subsection{Stability Analysis}

Looking at the location the eigenvalue's of LMPC and checking whether they are within unit circle we can decide the stability of the system. We also make a comparison with those of the optimal Discrete Linear Quadratic Regulator (DLQR) system. To grant stability as explained previous section we use $\alpha=1.6$, $\mathrm{N}_{\mathrm{p}}=200$ and $\mathrm{N}=8$.

In Figure 4, we can see that all the eigenvalues appear inside the unit circle as required. In addition, it is noted that the eigenvalues of the LMPC coincides with that of the optimal DLQR. This not only shows the system is stable but also shows that the controller performs optimally. The exponentially weighted cost function removes the problem of stability (ill numerical condition) because the model used in the prediction is changed to be stable using the scaling factor $\alpha$. As a result, the prediction horizon $N_{\mathrm{p}}$ can be selected to be sufficiently large without creating ill-conditioning (here $\mathrm{N}_{\mathrm{p}}=200$ ). Hence, closed-loop stability is guaranteed.

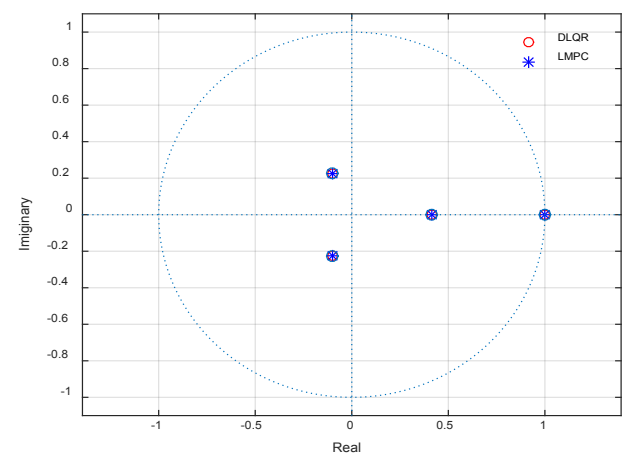

Figure 4. Eigenvalues for DLQR and LMPC

\subsection{Performance of LMPC as compared to SS-MPC}

In this section, we examine at the performance of LMPC when used to regulate the output voltage to reference signal. Also, LMPC is compared to SS-MPC. 
Here also we considered two cases:

(a) Performances comparison of Laguerre based Model predictive control(LMPC) and state space model predictive control(SS-MPC) simulated for $\mathrm{N}_{\mathrm{c}}=10 ; \mathrm{N}=2, \mathrm{Q}_{\mathrm{y}}=1$ and $\mathrm{R}=1$. Using these parameters, the closed-loop poles of the DLQR system are $-0.3815 \pm$ j0.3798, 0.4333 and 0 with feedback gain:

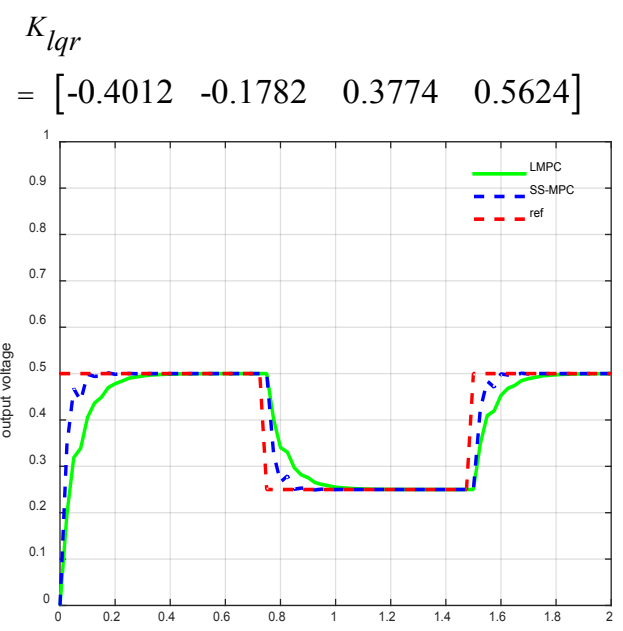

(a)

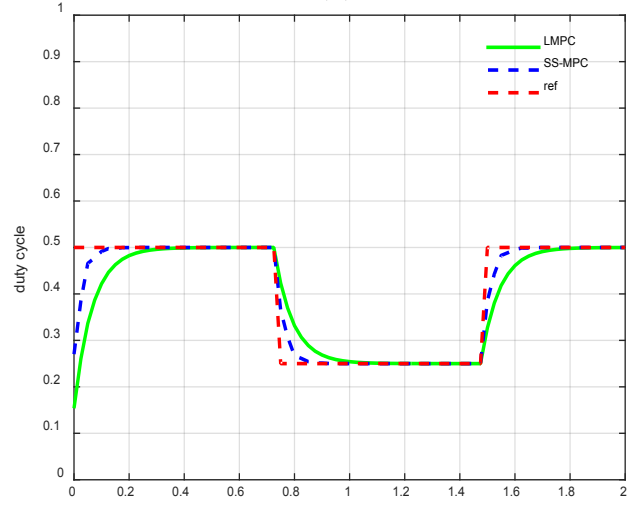

(b)

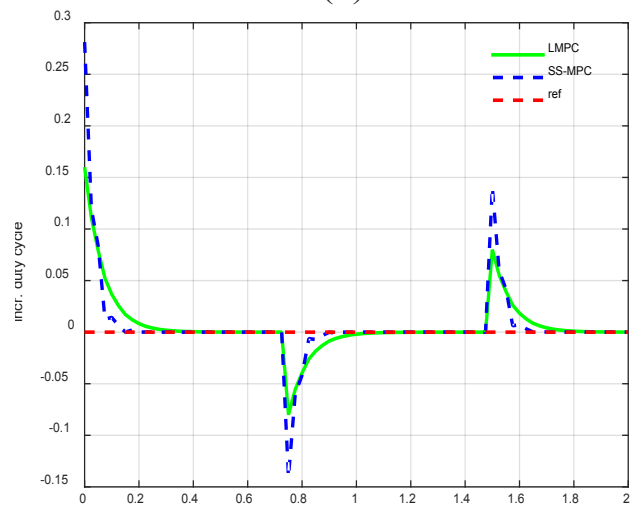

(c)

Figure 5. Simulation results for reference voltage variations $\mathrm{v}_{\mathrm{o} \text {,ref }}: 10-5-10 \mathrm{~V}$ obtained using LMPC and $\mathrm{SS}-\mathrm{MPC}$ for $\mathrm{N}=2$

(a) Output voltage $v_{0}$; (b) Duty Cycle d(t);

(c) Incremental duty cycle $\Delta d(t)$. (b) Performances comparison Laguerre based Model predictive control(LMPC) and state space model predictive control(SSMPC) simulated for $\mathrm{N}_{\mathrm{c}}=10 ; \mathrm{N}=5, \mathrm{Q}=1$ and $\mathrm{R}=1$. Using these parameters, the closed loop poles of the DLQR system are $-0.3815 \pm$ j0.3798, 0.4333 and 0 with feedback gain:
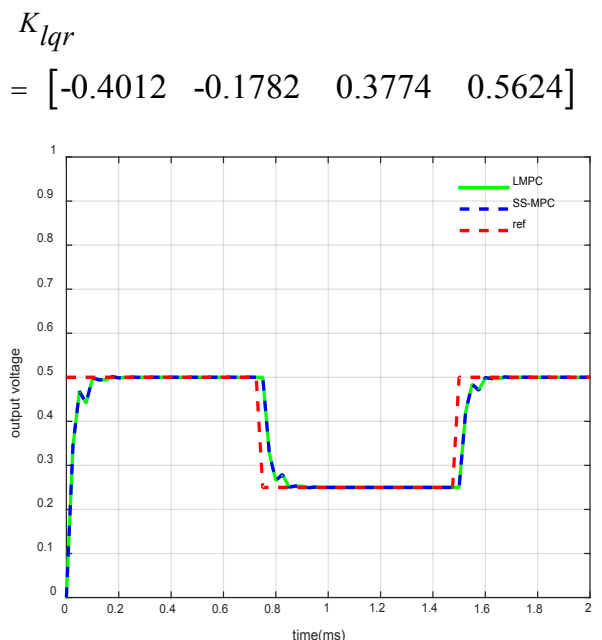

(a)

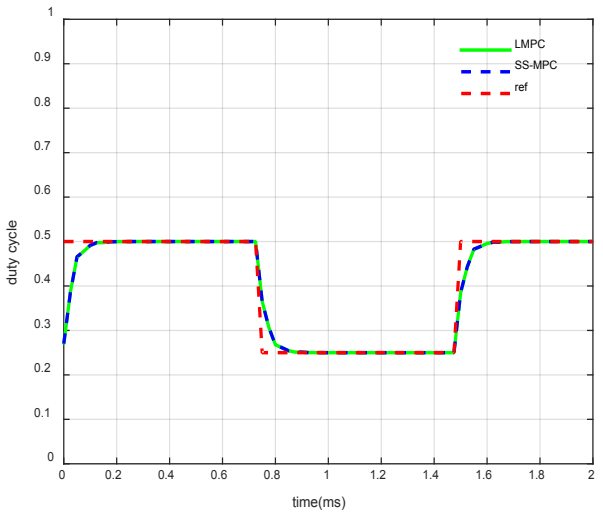

(b)

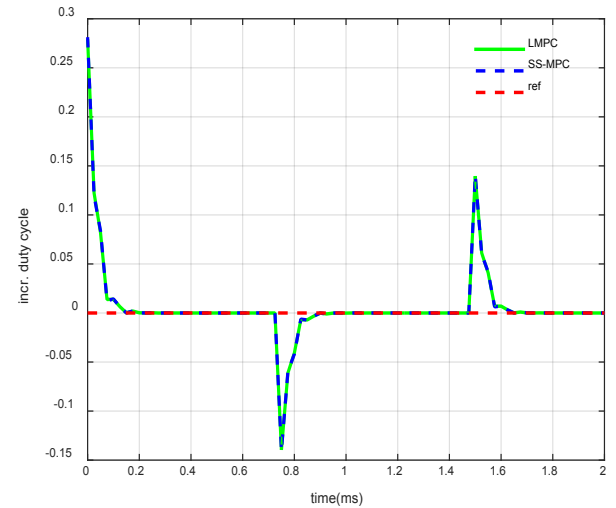

(c)

Figure 6. Simulation results for reference voltage variations $\mathrm{v}_{\mathrm{o}, \mathrm{ref}}: 10-5-10 \mathrm{~V}$ obtained using LMPC and $\mathrm{SS}-\mathrm{MPC}$ for $\mathrm{N}=5$

(a) Output voltage $v_{0}$; (b) Duty Cycle d(t);

(c) Incremental duty cycle $\Delta \mathrm{d}(\mathrm{t})$. 
As it can be seen from figure 5, SS-MPC performs better than LMPC. This is because the LMPC controller used lower Laguerre order $(\mathrm{N}=2)$.

In Figure 6, both LMPC and SS-MPC regulate the output voltage to the reference very well. However, LMPC performs better than SS-MPC in that it requires only five parameters $(N=5)$ to for the regulation compared to SS-MPC's minimum of $10\left(N_{c}=10\right)$ parameters. Thus, we can choose a Laguerre network with lower number of terms $N$ (that gives lower computation burden). The computational cost is lower if a smaller number of parameters are used.

\section{Conclusion}

Optimal control of the DC-DC buck converter was considered. We have presented state space averaging model and control approach for switchmode buck DC-DC converters by formulating a constrained optimal predictive control problem. The proposed predictive controller uses orthonormal Laguerre functions to capture control signal which reduces computation largely in real time. Also, exponential data weighing is used to reduce numerical issue, particularly with large prediction horizon. A quadratic objective function equivalent the one used in discrete linear quadratic regulator (DLQR) has been used. In stability analysis LMPC has been compared to the optimal DLQR system. The LMPC gives extra advantage as compared to the more commonly used MPC(SS-MPC) that it can handle the buck DC-DC converter control where rapid sampling and more complicated process dynamics are required. Simulation results have been given to show that the proposed controller guided to a closed-loop system. Finally, it has been shown that the output voltage has been tracked to the reference.

\section{Acknowledgements}

This research was supported by the Brain Research Program through the National Research Foundation of Korea (NRF) funded by the ministry of science, CT and Future Planning (NRF-2017M3C7A1044815).

\section{REFERENCES}

1. Čuk, S. \& Middlebrooks, D. (1997). A general unified approach to modelling switchingconverter power stages, J. Electron. Theor. Exp, 42(6), pp. 521-550.

2. Alvarez-Ramirez, J., Cervantes, I., Espinosa-Perez, G., Maya, P. \& Morales, A. (2001). A stable design of PI control for DC-DC converters with an RHS zero, IEEE Trans. Circuits Syst. I, 48(1), pp. 103-106.]

3. Aitouche, A., LI, S., Tian, Y. \& Wang, H. (2016) .Intelligent Proportional Differential Neural Network Control for Unknown Nonlinear System, Studies in Informatics and Control, 25(4), pp. 445-452.

4. Garofalo, F., Marino, P., Scala, S. \& Vasca, F. (1994). Control of DC-DC converters with linear optimal feedback and nonlinear feedforward, IEEE Trans. Power Electron, 9(6),pp. 607-615.

5. Mohan, N., Robbins, P. and Undeland M. (1989). Power Electronics: Converters Applications and Design,Wiley.

6. Chong, K., Yu, G. (2012). The Explicit Constrained Min-Max Model Predictive Control of a Discrete-Time Linear System with Uncertain Disturbances. IEEE Trans. on Automatic Control, 57(4), pp. 2373-2378

7. Wang, L., (2004). Discrete model predictive control design using Laguerre functions, Journal of Process Control, pp. 131-142.

8. Wang, L., (2001). Use of exponential data weighting in model predictive control design, In Proceedings of the 40th IEEE Conference on Decision and Control, $\left.D C^{\prime} 01\right)$, pp. 4857-4862.

9. De Belie, M., De Gussem'e, K., Melkebeek, J., Van den Bossche, A. \& Van de Sype (2006). Small-signal z-domain analysis of digitally controlled converters, IEEE Trans. Power Electron, 21(2), pp. 470-478.

10. Maksimovic, D. \& Zane, R. (2007). Smallsignal discrete-time modeling of digitally controlled PWM converters", IEEE Trans. Power Electron,22(6), pp. 2552-2556. 
11. Bloom, G. \& Severns, R. (1985). Modern DC-To-DC Switch mode Power Converter Circuits. Van Nostrand Reinhold.

12. Wang, L. (2009). Model Predictive Control Design and Implementation Using MATLAB, Advances in Industrial Control, Springer.

13. Anderson, B. \& Moore J. (1971). Linear Optimal Control, Prentice-Hall.
14. Wang, L. (2001). Discrete time model predictive control design using Laguerre functions, In Proceedings of the American Control Conference, pp. 2430-2435.

15. Wills, A. (2004). Technical report EE04025Notes on linear model predictive control. 